\title{
Dancing around the divisome: asymmetric chromosome segregation in Escherichia coli
}

\author{
Xindan Wang, ${ }^{1}$ Christophe Possoz, ${ }^{1}$ and David J. Sherratt ${ }^{2}$ \\ Department of Biochemistry, University of Oxford, Oxford OX1 3QU, United Kingdom
}

\begin{abstract}
By simultaneously tracking pairs of specific genetic regions and divisome proteins in live Escherichia coli, we develop a new scheme for the relationship between DNA replication-segregation, chromosome organization, and cell division. A remarkable asymmetric pattern of segregation of different loci in the replication termination region (ter) suggests that individual replichores segregate to distinct nucleoid positions, consistent with an asymmetric segregation of leading and lagging strand templates after replication. Cells growing with a generation time of $\mathbf{1 0 0} \mathrm{min}$ are born with a nonreplicating chromosome and have their origin region close to mid-cell and their ter polar. After replication initiation, the two newly replicated origin regions move away from mid-cell to opposite cell halves. By mid-S phase, FtsZ forms a ring at mid-cell at the time of initiation of nucleoid separation; ter remains polar. In the latter half of $\mathrm{S}$ phase, ter moves quickly toward mid-cell. FtsK, which coordinates the late stages of chromosome segregation with cell division, forms a ring coincident with the FtsZ ring as $\mathrm{S}$ phase completes, $\sim 50 \mathrm{~min}$ after its initiation. As ter duplicates at mid-cell, sister nucleoid separation appears complete. After initiation of invagination, the FtsZ ring disassembles, leaving FtsK to complete chromosome segregation and cytokinesis.
\end{abstract}

[Keywords: Chromosome segregation; divisome; FtsK; FtsZ; ori; ter]

Supplemental material is available at http://www.genesdev.org.

Received April 1, 2005; revised version accepted July 21, 2005.

Understanding of how bacterial chromosomes are organized and how this organization relates to replication, recombination, chromosome segregation, and cell division has emerged in recent years (for review, see Harry 2001; Margolin 2001; Draper and Gober 2002; Sherratt 2003; Espeli and Marians 2004; Lesterlin et al. 2004; Bates and Kleckner 2005). Importantly, this understanding has been informed by the exploitation of microscopic imaging of specific genetic regions, and of divisome and cytoskeletal structures using fluorescent fusion proteins, FISH (fluorescent in situ hybridization), and immunocytochemistry. However, the interpretation of many of these studies has been complicated by the fact that fastgrowing cells in which multiple rounds of replication are ongoing at the same time, and which therefore have overlapping $\mathrm{S}$ and G2/M phases, have been analysed. Furthermore, in most studies, a single genetic locus or protein was examined at any one time, and integration of data for multiple loci required combining results from different genetic backgrounds, growth conditions, and

\footnotetext{
${ }^{1}$ These authors contributed equally to this work.

${ }^{2}$ Corresponding author.

E-MAIL sherratt@bioch.ox.ac.uk; FAX 44-1865-275-297.

Article and publication are at http://www.genesdev.org/cgi/doi/10.1101/ gad.345305.
}

methodologies. Finally, genetic studies have been hampered because mutants with defects in either chromosome organization or segregation all have pleiotropic phenotypes that influence many aspects of DNA metabolism and growth. A current challenge is to use a combination of reagents and techniques that interfere minimally with cellular processes in order to gain a comprehensive and consistent picture of how chromosome organization relates to replication-recombination, and how these relate to chromosome segregation and cell division.

The 4.6-Mbp Escherichia coli circular chromosome is compacted $>1000$-fold into a compact nucleoid that occupies much of the central part of the cell. It is replicated bidirectionally from a unique origin, with replication termination occurring in a broad terminus region (ter) diametrically opposite from the origin. A series of studies using either FISH or the binding of fluorescent repressors to arrays of their cognate binding sites has demonstrated that specific genetic loci occupy specific positions within the nucleoid and cell, and that these change during the cell cycle (Glaser et al. 1997; Gordon et al. 1997; Niki and Hiraga 1998; Niki et al. 2000; Roos et al. 2001; Li et al. 2002, 2003; Lau et al. 2003; Bates and Kleckner 2005). Some studies have suggested that the ori 
and ter regions form $\sim 1-\mathrm{Mbp}$ macrodomains that act as independent units (Niki et al. 2000; Valens et al. 2004), although recent work in both E. coli and Caulobacter crescentus has suggested that loci $\sim 200 \mathrm{~kb}$ apart can occupy separate positions in the cell and segregate independently (Fekete and Chattoraj 2005; Viollier et al. 2004). Recent estimates of functional supercoiled domain size suggest that it may be as small as $10 \mathrm{~kb}$ (Deng et al. 2004; Postow et al. 2004).

A range of studies has proposed that the process of DNA replication is confined to a specific region of the cell, with unreplicated chromosomal DNA being reeled into the replication machinery and newly replicated DNA expelled from it (Lemon and Grossman 2000, 2001; Lau et al. 2003; Viollier and Shapiro 2004). Several mechanisms have been proposed to drive the segregation of DNA after replication: from replication itself, transcription (Dworkin and Losick 2002), and the action of actin-like cytoskeletal elements (Kruse et al. 2003; Soufo and Graumann 2004; Gitai et al. 2005), to the coordinate transcription of membrane proteins and their insertion into membrane ("transertion") (Woldringh 2002; Rocha et al. 2003). DNA sequences in E. coli, Bacillus subtilis, and $C$. crescentus that facilitate the segregation of newly replicated ori regions have also been described (Lin and Grossman 1998; Ben-Yehuda et al. 2003; Wu and Errington 2003; Fekete and Chattoraj 2005; Yamaichi and Niki 2004; Gitai et al. 2005).

After termination of replication, the two sister chromosomes need to be physically separated before segregation and cell division can be completed. Two potential impediments to such separation are catenation of the two monomeric sister chromosomes, and chromosome dimer formation by crossing over during homologous recombination (for review, see Espeli and Marians 2004; Barre and Sherratt 2005). Topoisomerase action is required for decatenation, while XerCD site-specific recombination at the recombination site dif, located in the ter region, converts dimers to monomers. FtsK, a 1329amino-acid integral membrane protein that localizes to the FtsZ ring functions directly in dimer resolution and facilitates decatenation by topoisomerase IV (Espeli et al. 2003). The 179-amino-acid N-terminal membrane domain of FtsK is required for cytokinesis, while the 500amino-acid C-terminal domain is a DNA translocase that functions in chromosome segregation and dimer resolution (Draper et al. 1998; Yu et al. 1998; Aussel et al. 2002). FtsK action may also facilitate chromosome segregation by organizing newly replicated ter regions at mid-cell (Lesterlin et al. 2004). Therefore, FtsK links chromosome segregation with cell division via its C- and N-terminal domains.

Localization of FtsK at the septum requires tubulinlike FtsZ, which plays a key role in cell division. FtsZ forms a multimeric ring at the division site and recruits other cell division proteins. The normal mid-cell positioning of FtsZ (and the additional quarter-cell positioning in rapidly growing cells) requires a functional Min system as well as a mechanism that directs the ring precisely to mid-cell at the appropriate time in the cell cycle (for review, see Margolin 2001; Wu and Errington 2004; Bernhardt and de Boer 2005).

In the work presented here, we have used live E. coli to simultaneously track pairs of fluorescent markers that include genetic regions and components of the divisome. Although some other work has simultaneously visualized two or more genetic regions in E. coli and C. crescentus (Niki et al. 2000; Fekete and Chattoraj 2005; Viollier et al. 2004; Bates and Kleckner 2005), previous studies have not compared gene position with that of divisome components. Cells growing with a generation time of $100 \mathrm{~min}$ at $37^{\circ} \mathrm{C}$, in which there is a single cycle of nonoverlapping G1, S, and G2/M phases were used. The results have allowed us to assemble a timeline for the E. coli cell cycle in which chromosome replication and segregation are related to the sequential assembly and disassembly of the divisome proteins FtsZ and FtsK. Furthermore, the segregation behavior of three different loci in the ter region leads us to propose a new model in which asymmetric chromosome segregation plays a central role in shaping chromosome organization.

\section{Results}

\section{Relative positioning of ori and ter}

Ori and ter regions were visualized in living cells by the binding of fluorescent CFP (cyan fluorescent protein) and YFP (yellow fluorescent protein) fusion derivatives of the LacI and TetR repressors to their respective operator arrays. We have fastidiously ensured that the reagents used to visualize and track components of the divisome and specific chromosomal loci close to the sites of initiation and termination of replication in living cells do not interfere significantly with the processes under analysis. Under the conditions used, binding of the repressors did not interfere with cellular growth rate, cell size distribution, or DNA replication as assessed by flow cytometry (Supplemental Material). This was achieved by expressing the repressors tandemly from a plasmid-borne weak constitutive promoter, and by reducing repressor binding by having an appropriate level of gratuitous inducer present (Lau et al. 2003). With this system, steady-state growth could occur while fluorescent repressors were being continually expressed and binding to chromosomal operator arrays. In all of the experiments reported here either a lacO or tet $O$ array (ori1) was placed $15 \mathrm{~kb}$ counterclockwise from the replication origin (oriC), while the ter region $1 a c \mathrm{O}$ or tet $\mathrm{O}$ array was placed $50 \mathrm{~kb}$ clockwise (ter3), $200 \mathrm{~kb}$ clockwise (ter2), or $200 \mathrm{~kb}$ counterclockwise (ter4) of the dif site in the ter region (Fig. 1A). A combination of snapshot and time-lapse microscopy of live cells was used to generate the data.

Snapshot micrographs of individual cells derived from an exponentially growing liquid culture at $37^{\circ} \mathrm{C}$ with a 100 -min generation time were binned into groups each containing a given arrangement of ori1 and ter2 loci (Fig. 1B). Seven major groups were identified on the basis of focus position and number. Then the likely sequential series of events representing the cell cycle was inferred 
A
C
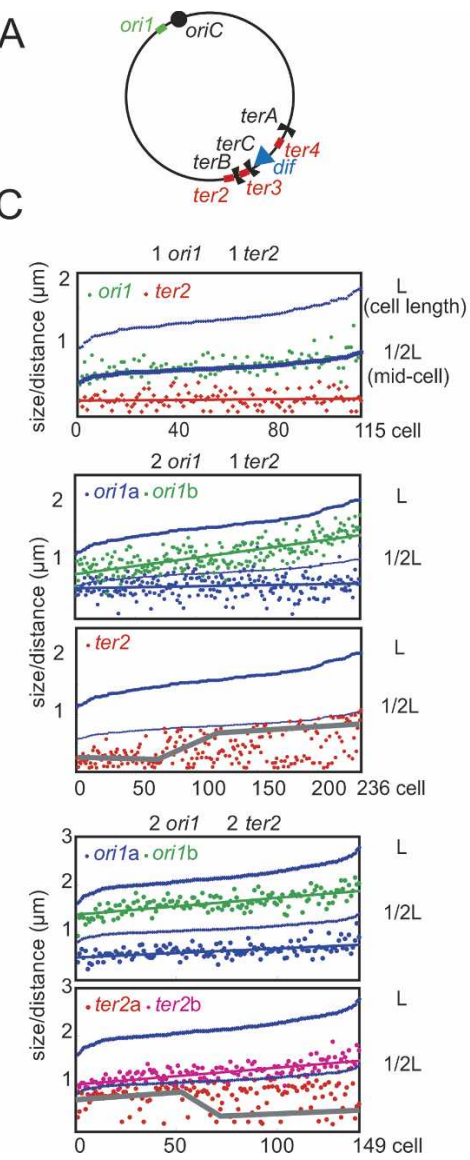

B

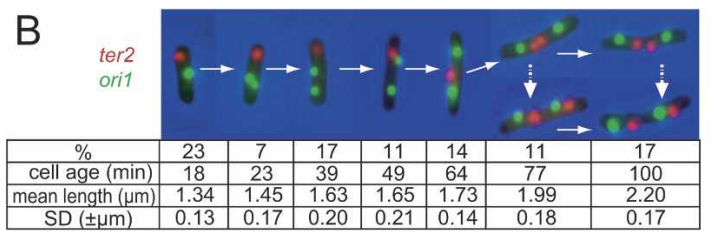

D
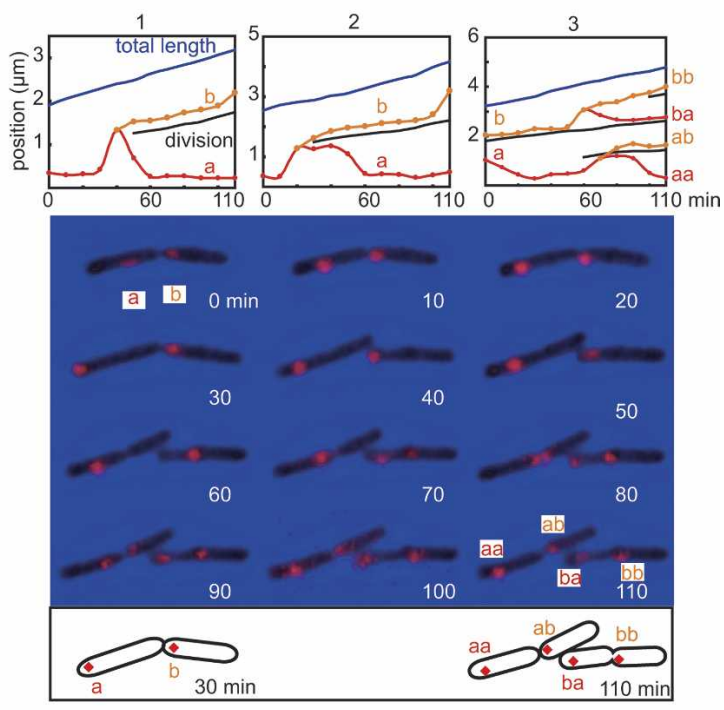

Figure 1. Simultaneous microsopic analysis of ori and ter regions. (A) Schematic of the circular 4.6-Mbp E. coli chromosome indicating the positions of the lacO and tetO arrays, which can be present interchangeably at all positions. Also shown are the major Tus-dependent replication termination sites $(\operatorname{ter} A$, ter $B$, and $\operatorname{ter} C)$; the origin of replication ori $C$; and dif, the site for chromosome dimer resolution. (B) Snapshots of ori1 (tetO)-ter2 (lacO) cells (500 analyzed), with fractions of the seven indicated groups and their conversion into fractions of cell cycle. The groups are as follows: I, polar ter2 and nonpolar ori1; II, polar ter2 and closely positioned sister ori1 foci in the region of mid-cell; III, polar ter2 and well separated sister ori1 foci; IV, ter2 positioned between pole and mid-cell and well separated sister ori1 foci; V, ter2 focus at mid-cell and well separated ori1 foci; VI, separated ter2 sister foci; VII, separated sister ter2 foci and initiation of invagination. Mean cell lengths and standard deviations (SD) for each group are indicated. White arrows indicate the inferred sequence of events. Thirty-three percent of the cells that have undergone ter duplication-separation (groups VI and VII) have one of the sister ter2 foci at the pole, consistent with movement of one of the newly replicated ter foci to the nucleoid edge (broken arrows). Cell age is the transition point from the indicated cell group to the next group. The data were obtained from a 100 -min generation time liquid culture growing exponentially at $\mathrm{A}_{600}=0.3$. (C) Distributions of ori1 and ter2 positions within individual cells (data set used for $B)$. Each cell is shown on the abscissa and its length focus position on the ordinate. (Top) (1× ori1 and $1 \times$ ter 2$)$ cells. (Middle) $(2 \times$ ori1 and $1 \times$ ter 2$)$ cells. $($ Bottom) $(2 \times$ ori1 and $2 \times$ ter2) cells. The middle and bottom panels have each been separated into two for clarity, with ori1 and ter2 positions shown separately. Cell length and mid-cell position for each cell is shown in blue. Best-fit lines of ori/ter position throughout the distribution for selected markers are indicated by thin red lines (ter) and green/blue lines (ori). In the $(2 \times$ ori1 and $1 \times$ ter 2$)$ and $(2 \times$ ori1 and $2 \times$ ter 2$)$ distributions, the gray lines are drawn to underline the movement of the ter array from the nucleoid edge to mid-cell (middle) and away from mid-cell back to the original pole (bottom). (D) Time-lapse progressions of asymmetric ter2 segregation. The upper panel shows ter2 position as a function of time in three different progressions that track ter2 duplication-segregation over at least one generation. Note the movement of polar ter2 to mid-cell, ter2 duplication, and relocation back to the original pole. The black line positions the division site when it is visible during invagination and the new poles after division. Sister ter2s are labeled by letters, and their positions in the cell are tracked by orange and red lines. The bottom panel shows the cells for time-lapse 3. Schematics of cells at 30 and 110 min are shown.

(Fig. 1B, white arrows). Estimates of mean cell length within each group supported the assignment to a sequential cell cycle, while time-lapse microscopy confirmed the assignment (see below; data not shown). The proportions of cells in each group were determined. Since relative cell number per age class shows an exponential de- cline from one (newborn cells) to 0.5 (cells completing division) in an exponential culture, the cumulative frequency of each cell type was converted into cell age for each transition from one group to the next (Fig. 1B; Skarstad et al. 1985; Aarsman et al. 2005). Assuming steady-state growth in the exponentially growing cul- 
ture, these data lead to the conclusion that sister ori1 focus separation initiates at $\sim 18 \mathrm{~min}$, while ter2 array separation $200 \mathrm{~kb}$ from dif initiates at $\sim 64 \mathrm{~min}$. Therefore, $S$ phase (the period of DNA synthesis) should be complete by $\sim 70 \mathrm{~min}$, and $\mathrm{G} 2 / \mathrm{M}$ (the period between completion of DNA synthesis and completion of cell division) will be at least $30 \mathrm{~min}$. Assuming a single complete replication cycle within a cell (Supplemental Material), G1 (the period before initiation of DNA synthesis) is $0-18 \mathrm{~min}$. Finally, S phase will be close to $50 \mathrm{~min}$ if any period between replication and focus separation is similar for the ori1 and ter2 loci, as suggested by the work of Bates and Kleckner (2005).

The primary data for the snapshots are shown in Figure 1C. The cell length and ori1-ter2 focus position for each of 115 cells ( $1 \times$ ori 1 and $1 \times$ ter 2$), 236$ cells $(2 \times$ ori 1 and $1 \times$ ter 2$)$, or 149 cells $(2 \times$ ori1 and $2 \times$ ter 2$)$ are shown. Each individual cell is displayed on the abscissa and its length and foci positions on the ordinate. For clarity, the data for $(2 \times$ ori 1 and $1 \times$ ter 2$)$ and $(2 \times$ ori 1 and $2 \times$ ter 2$)$ cells are separated to show the ori1 and ter2 positions separately. The polar location of ter2 and central location of ori1 prior to replication is evident (Fig. 1C, top panel). We note that initial ori1 separation appears to be asymmetric, with the ori1 distal to the ter2 array (ori1a), achieving its quarter position before the ori1 proximal to the ter2 array (ori1b). This asymmetry is evident in the selected snapshots (Fig. 1B). An asymmetry of oriC segregation has been reported in a FISH analysis of $E$. coli cells growing at similar growth rates (Bates and Kleckner 2005). Although ter2 movement from the pole to midcell initiates $\sim 21$ min after ori1 separation in most cells, the timing of ter2 movement to mid-cell is heterogeneous, with some ter2 arrays still polar late in S phase (Fig. 1C, middle panel). In the cells that have duplicated ter2, the quarter positions of the sister ori1 foci are evident, as is the asymmetric positioning of many of the sister ter2foci (Fig. 1C, bottom panel).

Analysis by flow cytometry (FACS) of cultures growing under the same conditions as for the snapshot analysis not only shows that the presence of the arrays and repressors do not interfere significantly with DNA metabolism, but provides support for our inferences from the snapshot analysis (Supplementary Fig. 1).

\section{Asymmetric segregation of the ter2 locus: polarization}

Within the cell population that had duplicated the ter2 array and were approaching cell division, 67\% of the cells had the two ter2 loci close to mid-cell, while $33 \%$ of cells had one of the two newly replicated ter2 arrays positioned at the edge of the nucleoid adjacent to a pole, while the other was close to mid-cell (Fig. 1B,C). We could not be sure from the snapshot analysis whether these represented alternative states/pathways or a sequential progression in which one of the newly replicated sister ter regions moves away from mid-cell to a pole (Fig. 1B, broken arrows).

Tracking of ter2 by time-lapse analysis confirmed that in most cell divisions one of the newly replicated sisters moved away from mid-cell to its original pole, while the other remains at mid-cell. When the fates of 10 polar ter2 loci were followed by time-lapse microscopy, seven showed asymmetric segregation of sister foci after duplication (Fig. 1D), although in one of these, after the initial asymmetric segregation, the newly replicated sister termini eventually positioned at opposite poles. The remaining three ter2 loci moved to mid-cell, duplicated, and the sisters remained at mid-cell. In the three latter cases, we do not know whether the sister ter2 loci would eventually segregate asymmetrically. This asymmetric segregation generates one daughter cell with ter2 adjacent to an old pole and the other with ter2 adjacent to a new pole. It is noteworthy that we expect the pattern of asymmetric segregation (polarization) to be heritable (see below).

\section{Relative timing of Fts $Z$ and FtsK ring assembly-disassembly and their positioning relative to ori-ter}

Next, the cytological information above was related to the timing of FtsZ and FtsK ring assembly and disassembly. A CFP derivative of FtsZ could not replace wild-type FtsZ and led to filamentation when overexpressed in a wild-type background. Therefore, in order to visualize FtsZ-CFP, an ectopic plasmid-born copy of the FtsZ-CFP gene was expressed constitutively from a weak plasmid promoter. Under the growth conditions used, cells expressing this protein along with wild-type FtsZ grew with normal length and division characteristics. Western blotting (Fig. 2A) showed that $\sim 11 \%$ of total FtsZ was FtsZ-CFP under these conditions, although we do not know if the FtsZ-CFP:FtsZ ratio in the rings is the same. Other studies with a fluorescent FtsZ derivative have reported normal localization and division when up to $50 \%$ of the cellular FtsZ is fluorescent. Furthermore, they have shown that FtsZ ring formation and disassembly in fast-growing E. coli were identical when fluorescent and immunocytochemical detection of FtsZ were compared (Sun and Margolin 1998, 2001; Thanedar and Margolin 2004).

A chromosomal derivative of the wild-type FtsK gene expressed from its normal promoter was tagged with YFP at the $\mathrm{C}$ terminus. This FtsK-YFP protein is functional as judged by its ability to support FtsK-dependent dimer resolution on a model plasmid substrate (Recchia et al. 1999) and the normal doubling time and cell size distribution of cells containing the derivative during exponential growth (data not shown). Nevertheless, flow cytometry indicated that $\mathrm{G} 2 / \mathrm{M}$ was somewhat extended in a fraction of cells, leading to a premature initiation of DNA synthesis. Therefore, FtsK-YFP is probably not completely functional, although the normal cell length distribution makes us confident that visible FtsK-YFP ring formation and disappearance is a reflection of wildtype FtsK ring formation and turnover.

Snapshots of FtsK and FtsZ localization with respect to each other and to ori-ter are shown in Figure 2B. They are validated by time-lapse progressions (Fig. 2C). From 

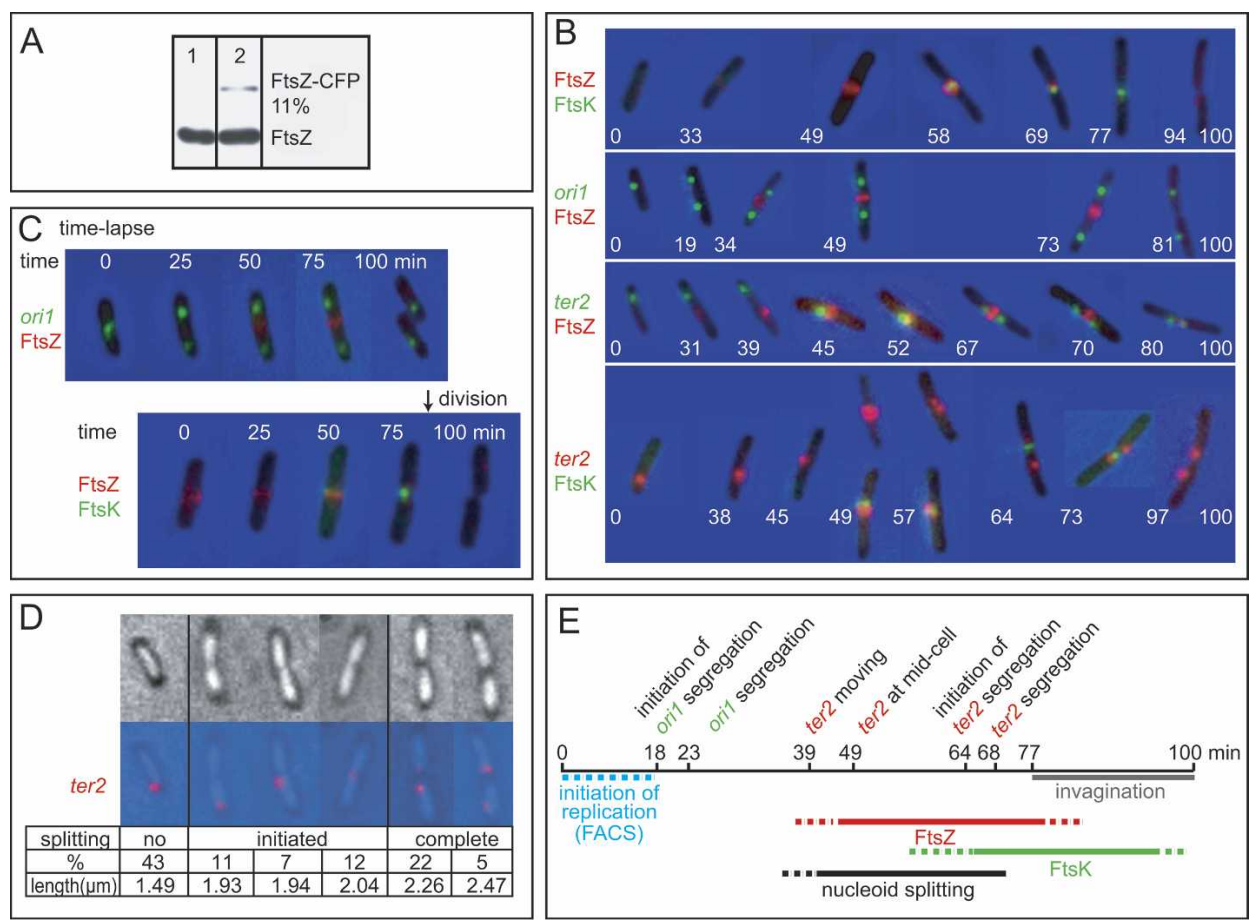

Figure 2. Relative positioning and timing of FtsZ and FtsK rings and their relationship to ori1-ter2 and the nucleoid. (A) Relative proportions of FtsZ and FtsZ-CFP in a cell expressing wild-type FtsZ from its endogenous chromosomal position and FtsZ-CFP from a weak plasmid-borne constitutive promoter, assayed by Western blots using a FtsZ polyclonal antibody. (Lane 1) Cell lysate from AB1157 cells. (Lane 2) Lysate from cells expressing FtsZ-CFP in the presence of endogenous FtsZ. (B) Relative positions of FtsZ, FtsK, and ori1-ter2 as judged by snapshot analysis. Analysis as in Figure 1B. Times (shown in white) are the computed times at which the indicated transitions occur, calculated from the abundance of each class. Two hundred to 500 cells were examined for each pairwise analysis. (C) Time-lapse analysis of FtsZ ring formation with respect to ori1 (top) and FtsK ring formation (bottom). The two progressions are aligned approximately with respect to relative cell age. Each progression is slightly less than one generation. The times of appearance of the FtsZ and FtsK rings with respect to ori1 separation, and the separation in time between FtsZ and FtsK ring formation, are in good agreement with the snapshot data $(B) .(D)$ Time of nucleoid splitting with respect to ter2 position. Cells are divided into three classes: no visible nucleoid splitting, nucleoid splitting initiated, and nucleoid splitting complete. Two of the classes are then subdivided depending on ter2 position. The fractions of cells in each class are given along with their mean length. These fractions are then converted into cell ages as before. This leads to nucleoid splitting being initiated at $\sim 35$ min and complete separation occurring at $\sim 66 \mathrm{~min}$ in 100-min generation time cells. (Top panel) Phase contrast. (Bottom panel) ter2 fluorescence superimposed on the phase-contrast image. (E) Timeline constructed using the snapshot data from Figures 1 and 2 and the flow cytometry data (Supplemental Material). Time-lapse progressions support the sequence of events.

these observations we conclude that FtsZ rings form in the middle of $S$ phase, with the initial FtsZ ring often being diffuse although centered at mid-cell; a tighter ring forms as $\mathrm{S}$ phase progresses. Visible FtsK rings form some 22 min after the FtsZ ring, close to the time of ter replication-duplication at the end of $S$ phase. FtsK ring formation does not appear to depend on completion of DNA replication, since they form in a fraction of cells prior to sister ter2 separation. Furthermore, delaying ter replication-segregation as a consequence of tight binding of repressor to an array did not prevent FtsK ring formation (data not shown). The FtsZ ring disassembles $\sim 20$ min after FtsK ring formation, leaving an FtsK ring that remains until the completion of cytokinesis some 18 min later. Thus, these two dynamic ring structures act at the septum at different times, but for similar periods.

The differential appearance of FtsZ with respect to FtsK reported here is in general accord with the findings of Aarsman et al. (2005), who observed a 20-min delay between FtsZ ring formation and FtsK-dependent localization of FtsQ. Furthermore, the same laboratory has presented evidence that cells completing cytokinesis have already disassembled their FtsZ ring (den Blaauwen et al. 1999). During the successive recruitment of divisome proteins to mid-cell, the major delay is between the formation of the FtsZ ring and the FtsK ring. This delay is in accord with the observation that fast-growing cells can have three FtsZ rings, but never more than a single FtsK ring (Margolin 2001; our unpublished data).

In order to relate the timing of the events recorded in Figures 1 and $2 \mathrm{~A}-\mathrm{C}$ to nucleoid separation, we examined ter foci with respect to nucleoids visualized by phase contrast microscopy in high refractive index medium (Fig. 2D; Mason and Powelson 1956; H. Niki, pers. comm.). We grouped nucleoids into three classes on the basis of visual inspection: no evidence of separation into two, partial separation into two, and complete separation into two. Completely separated nucleoids became 
evident only at the time of ter segregation, while partial nucleoid separation initiated in the middle of $S$ phase (Fig. 2D). We then observed FtsZ-CFP rings in cells whose nucleoids were visible in high refractive index medium (data not shown). The FtsZ ring forms at midcell close to when nucleoid separation is first observed: $72 \%$ of cells that had initiated nucleoid separation had an FtsZ ring, while $4 \%$ of cells with no discernable nucleoid splitting had an FtsZ ring. We cannot be sure whether nucleoid separation had initiated, but was not observed, in this minority class. The results show that the FtsZ ring initially forms some 20-25 min before nucleoid separation is complete, when there is still a substantial concentration of DNA in the region of the nucleoid over which the $\mathrm{Z}$ ring forms.

The complete data, along with those from Figure 1B, are summarized in a timeline (Fig. 2E). In summary, the results in Figures 1 and 2 show that at a 100-min generation time, cells are born with ter close to a nucleoid edge adjacent to a pole, while ori is close to mid-cell. Newly replicated sister ori foci initiate separation at 18 min, moving from mid-cell to opposite cell halves soon after, and eventually reaching the quarter positions. This movement apart toward the quarter positions is at least three times faster than the rate of cell elongation and maybe as much as 15 times faster (Figs. 1B-D, 2C,E). Initiation of DNA replication occurs in the first $18 \mathrm{~min}$ after birth. An FtsZ ring forms at mid-cell at about the time initiation of nucleoid splitting is observed, $\sim 25 \mathrm{~min}$ before ter2 duplication initiates. As $\mathrm{S}$ phase progresses, the ter marker moves to mid-cell, reaching it in the latter half of $S$ phase. This movement can occur in a time less than the interval between two time-lapse images separated by $10 \mathrm{~min}(>0.2 \mu \mathrm{m} / \mathrm{min}, 10-20$ times faster than the rate of cell elongation) (Fig. 1D). Separation of the two sister ter markers occurs close to mid-cell. After ter array duplication and prior to most cell divisions, one of the two ter arrays remains close to mid-cell while the other moves to the distal nucleoid edge adjacent to a pole at a rate of $>0.1 \mu \mathrm{m} / \mathrm{min}, 5-10$ times faster than the rate of cell elongation (Fig. 1D).

The precise timings presented in the timeline (Fig. 2E) assume the exponential population is in steady state. If there were any heterogeneity in the population, for example, a fraction of newborn cells that is quiescent in G1, it would not change the sequence of proposed events, but would influence the precise event timings.

\section{Independent positioning and separation of pairs of loci} in the ter region

Use of any one of the three ter loci showed a similar pattern of asymmetric segregation and polarized localization. Newborn cells had the locus at or close to a nucleoid edge adjacent to a pole and the locus positioned at mid-cell in the latter stages of S phase, with replication occurring close to mid-cell. When we examined the simultaneous positioning of ter2-ter3 loci separated by $150 \mathrm{~kb}$ on the same replication arm (replichore), we noted from snapshot analysis that the loci did not nec- essarily colocalize. The most frequent classes of cells were ordered to show the inferred sequence of events (Fig. 3). This sequence of events was confirmed by timelapse photography (data not shown). Cells with the two loci colocalizing at a pole had the shortest length, whereas cells with one polar focus and one focus positioned closer to mid-cell were longer and had ter2 closer to mid-cell, consistent with the expectation that ter2 moves to mid-cell in order to replicate before ter3. Similarly, ter2 duplication at mid-cell precedes ter3 duplication, as does sister ter2 repositioning at a pole.

We conclude that the individual chromosomal domains that contain these loci behave independently, both temporally and spatially, moving independently to mid-cell prior to replication, replicating independently, and segregating away from mid-cell after replication. This is consistent with recent estimates of a $\sim 10-\mathrm{kb}$ chromosome domain size in E. coli (Postow et al. 2004).

Our observations on differential ter array positioning and segregation, along with our inferences regarding the relationship between initiation of DNA replication and ori1 focus separation, provide no evidence for periods of sister cohesion that extend for much of the cell cycle for either the ori or ter regions, although we would not have identified periods of cohesion of $<15 \mathrm{~min}$. In the ter region at least, any cohesion that there is must be broken in small stretches as segregation proceeds, because the ter2-ter3 markers $150 \mathrm{~kb}$ apart segregate independently over time as well as in space. This observation is consistent with the demonstration that pairs of fluorescent $E$. coli ori markers $233 \mathrm{~kb}$ apart position and duplicate independently (Fekete and Chattoraj 2005), while in C. crescentus a series of genome-wide fluorescent markers 125-250 kb apart segregate sequentially and independently after replication (discussed in Breier and Cozzarelli 2004; Viollier et al. 2004). Also consistent with our observations is the report that $E$. coli ori $C$ and ter remain cohesed for 15-20 min after replication, although two other markers, one just to the right of oriC and the other midway between oriC and ter, appear to remain cohesed

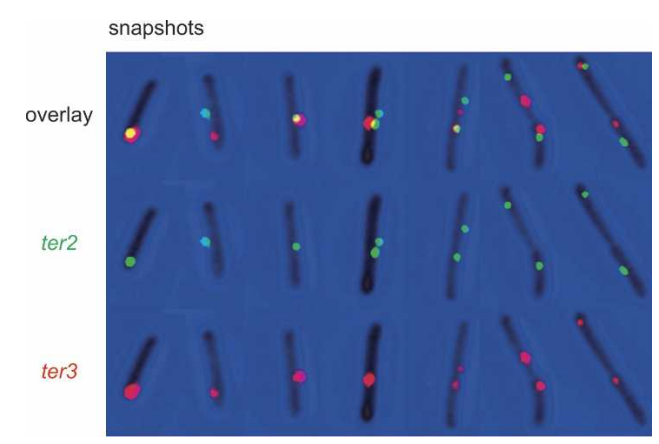

Figure 3. Independent positioning and segregation of different marker arrays in the ter region. Snapshots simultaneously visualizing ter 2 and ter3, separated by $150 \mathrm{~kb}$ on the same chromosome arm (replichore). Cell length increases from left to right, as does the inferred sequence of events in which the ter2 marker (green) moves from pole to mid-cell first, duplicates first, and segregates away from mid-cell first, consistent with its expected replication by the counterclockwise fork. 
after replication for a substantial proportion of the cell cycle (Bates and Kleckner 2005).

Early FISH experiments with E. coli led to the idea of a 1-Mbp ter macrodomain (Niki et al. 2000). Subsequent experiments, in which a fluorescent ParB fusion protein binding to a range of ter-located parS sites was used to visualize the E. coli ter region, also were used to support the idea of a single ter domain, albeit somewhat smaller (Li et al. 2003). In contrast, the independent spatial and temporal positioning-segregation of ter2-ter3 reported here gives no indication of such a ter domain. We note that the earlier FISH experiments, although pioneering in their time, neither simultaneously positioned pairs of markers $\sim 200 \mathrm{~kb}$ apart in the ter region, nor had high spatial resolution. Again, in the latter study, only a single locus was examined in any one strain, while the very late duplication-segregation of the ter foci makes us suspect that ParB-GFP bound to parS may have delayed duplication-segregation as a consequence of its "spreading" and silencing properties (Rodionov et al. 1999; our unpublished data).

\section{The pattern of asymmetric segregation is replichore dependent}

In order to understand more about the asymmetric segregation of ter arrays and how this leads to a polarized arrangement of sister nucleoids, we tracked the segregation of ter arrays singly and in pairs, in filaments induced after cephalexin treatment. The rationale for this was that within a given filament, the replication-segregation cycles of individual chromosomes should be synchronized, and the lineages of sister nucleoids can be tracked experimentally from the time of inhibition of division.

The asymmetric segregation of the single ter2 locus was dramatic: $97 \%$ of informative sister nucleoids pairs exhibited asymmetric segregation of newly replicated sister ter2 foci. Furthermore, in filaments containing four or eight nucleoids, the majority had the asymmetric pattern polarized throughout the filament, with each asymmetric segregation directing one sister ter2 away from mid-cell in the same direction $176 \%$ of groups of four nucleoids related by immediate descent ["cousins"] maintained polarization for two generations within the filament) (Fig. 4A). Rarely, both newly replicated sister foci segregated away from mid-cell in a nucleoid pair, or the polarity of asymmetric segregation reversed at the center of a filament.

When we simultaneously examined ter2-ter3, which are located on the same replichore and which are expected to be replicated by the counterclockwise replication fork since clockwise forks will normally be halted at terC $\sim 50 \mathrm{~kb}$ before ter3 (Fig. 1A), the majority pattern (94\% of informative sister nucleoids) had the asymmetric segregation of ter2 and ter3 directed toward the same pole (isopolarization) (Fig. 4B).

In contrast, when we simultaneously examined ter2ter4 loci located on different replichores, $200 \mathrm{~kb}$ to either side of dif, the major pattern $164 \%$ of informative sister nucleoids) was equally dramatic. Each locus alone dis- played the asymmetric segregation observed previously, but the two loci exhibited an opposed pattern; i.e., if one of the sister ter2 foci of each pair segregates to the "left", one of the sister ter4 foci segregates to the "right" (Fig. 4B). We believe this "opposed" yet complementary behavior (opposite polarization) is a consequence of the two loci being replicated by different forks (see below and Fig. 4C for discussion). A substantial minority of filaments $(28 \%$ of sister nucleoids) cosegregated the ter2-ter4 loci, as for ter2-ter3 (Fig. 4B). We believe this arrangement results when ter2-ter 4 are replicated by the same counterclockwise fork; this is expected to occur relatively frequently since the first replication barrier to the counterclockwise fork is distal to ter4 (terA) (Fig. 1A). More rarely we saw one of two symmetric patterns. In one, both sisters of a given locus remain at mid-cell, while each sister of the other locus moved toward opposing poles; in the other, all four foci moved to opposite poles (Fig. 4B). This rare symmetric segregation pattern will still generate sister nucleoids that maintain leftright orientation, although this behavior shows that the sister leading and lagging strand templates can be addressed rarely to symmetrical rather than asymmetric positions (see below).

The polarized pattern of ter segregation in the cephalexin filaments is consistent with our observation that in exponential cells, one of the newly replicated ter arrays moves toward the pole previously occupied by the mother ter (Fig. 1D). Therefore, the polarized asymmetric segregation is not an artifact of cephalexin treatment and is not influenced by the adjacency of a cell pole. Together, the results suggest that each given template is addressed to a specific heritable location after replication. We conclude that the pairwise patterns of asymmetric ter segregation are directed by which replication fork duplicates a given marker, and propose below that this behavior is general for all chromosome markers.

\section{Discussion}

New insight into how replication directs chromosome organization

The results presented here demonstrate a remarkable and unexpected heritable asymmetry of ter marker segregation that is polarized throughout filaments. This pattern of segregation leads us to propose a new model in which replication and chromosome segregation direct chromosome organization (Fig. 4C).

In 100-min generation time cells with sequential and nonoverlapping G1, S, and G2/M phases, a nonreplicating chromosome has its origin region at mid-cell with "left" (Fig. 4C, red) and "right" (Fig. 4C, blue) replichores positioned side by side, ter2 adjacent to the left pole, and ter4 adjacent to the right pole (Fig. 4C). Late-replicated DNA (the ter region) is on the outside of the nucleoid, while ori (Fig. 4C, unfilled circles) and other early replicating DNA is close to mid-cell. In a cell just prior to division, the replicore arrangement is "left-right-septum-left-right" and in a filament is "left-right-left- 
Wang et al.

A

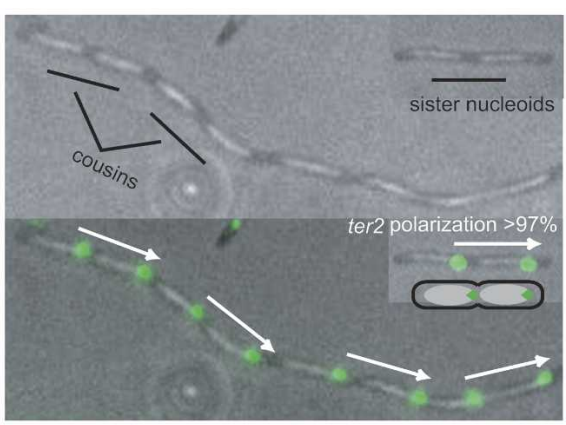

B

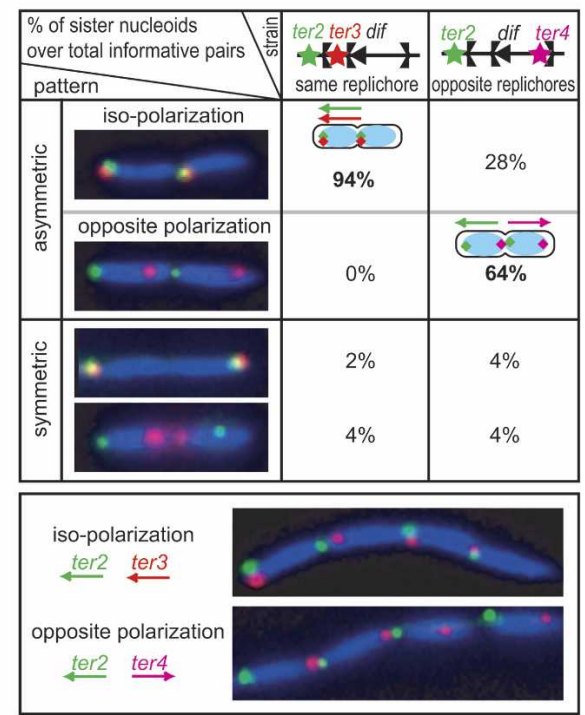

C
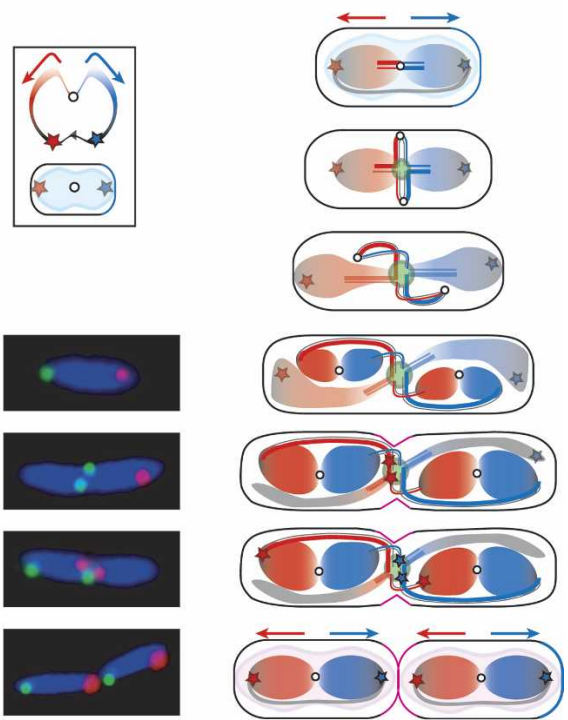

Figure 4. Asymmetric segregation of the ter region. (A) Positioning of ter2 in cephalexin filaments. Nucleoids are visualized in gelatin using phase contrast and the ter2 array by fluorescence. Polarized asymmetric segregation (arrows) accounts for $97 \%$ of informative sister nucleoid pairs within filaments. Informative nucleoid pairs are those related by immediate descent in which ter2 has duplicatedsegregated (160 of 222 nucleoid pairs were informative). (B) Relative positioning of ter2-ter3 and ter2-ter4 in cephalexin filaments. Nucleoids are stained with DAPI (blue). Four patterns of informative sisters were observed: two asymmetrical patterns (iso- and opposite polarization), accounting for $>90 \%$ of informative sisters, and two symmetrical patterns. Informative nucleoid sister pairs are those in which both foci had duplicated and at least one sister derived from a given locus had segregated away from mid-cell (46 of 437 ter2-ter3 and 26 of 63 ter2-ter4 nucleoid pairs were informative). The bottom panel shows two examples of polarized segregation in four-nucleoid filaments. $(C)$ Model for the relationship between replication-segregation and chromosome organization. Left and right replichores are colored red and blue, respectively. Ter2 is shown as a red star and ter4 as a blue star. (Left panel) Schematic of the bacterial chromosome and a cell containing that chromosome is shown boxed at the top, while representative snapshots of cells with ter2-ter4 are shown below. (Right panel) Schematic of cells going from G1 to G2/M (top to bottom). The origin of replication is shown as an unfilled circle and the replication machinery as a green circle at mid-cell. Newly replicated DNA is less pale than parental DNA, which loses color as replication proceeds. The schematic shows ter2 moving to mid-cell and replicating before ter4. A region of ter DNA links the outer nucleoid edges (black line). See text for further details.

right-left-right-left-right...", consistent with the ter data from exponentially growing cells and from cephalexin filaments. Note that in the nonreplicating chromosome, the ter2 and ter4 loci regions are connected directly by a DNA segment that passes from one nucleoid edge to the other. Since dif can be replicated by either the clockwise or counterclockwise fork, dif could be on either nucleoid edge or positioned anywhere between the two edges, results that are consistent with FISH data (Niki et al. 2000).

We propose that the asymmetric ter segregation reflects asymmetric segregation of all loci after initial oriC segregation away from mid-cell. A segregation mecha- nism that directs leading strand (Fig. 4C, thicker red and blue lines) and lagging strand (Fig. 4C, thinner red and blue lines) templates at each fork to different cellular addresses is the obvious way of explaining the asymmetric segregation pattern (Fig. 4C, middle panels). We have arbitrarily chosen to segregate the leading strand template sisters toward the outer nucleoid edge and the lagging strand templates toward the nucleoid center. The heritable pattern of asymmetric ter segregation must ensure that the left replichore is directed to the "left" and the right replichore to the "right" after replication. A model of strand-specific chromosome segregation, consistent with that here, has been proposed on the basis of 
how highly expressed genes may segregate heritably to "hyperstructures" (Rocha et al. 2003).

A key feature of the model is that as replication proceeds there is a sequential layering of new DNA on either side of the sister ori regions, left replichore on the left and right replichore on the right. In the middle of $S$ phase the reeling in of unreplicated DNA toward the replication machinery at mid-cell (Fig. 4C, green circle) and the continuing placement of newly replicated leading strand DNA toward the pole causes the unreplicated ter loci to move in from the nucleoid edge toward midcell.

The observation that both ori and ter foci duplicate in the region of mid-cell is consistent with other data and models which propose that replication is localized to the mid-cell region (Glaser et al. 1997; Gordon et al. 1997; Koppes et al. 1999; Lemon and Grossman 2000, 2001; Li et al. 2002; Lau et al. 2003). Our data do not address the question of whether the replication machinery forms on positioned oris or whether positioned replication machinery acts to localize ori. If there is any active positioning of ori at the quarter positions it is likely to require interaction with protein components. In such a case it is conceptually simpler to have prepositioned replication machinery localizing ori, consistent with a recent report in which positioning of DnaX, a component of the replication machinery, precedes ori positioning (Bates and Kleckner 2005). The position of ori close to mid-cell in newborn cells reflects the prior position of the two ori regions close to the quarter positions in the progenitor mother cell. Although we have previously suggested that an uncharacterized positional "mark" may be used to place the ori at the quarter position in a cell after its duplication (Lau et al. 2003; Sherratt 2003), the model here demands only that sister ori regions move away bidirectionally from mid-cell after replication. Any initial sister ori separation from mid-cell will eventually be reinforced by a segregation mechanism that places left and right replichores on each side of sister oriC regions as replication proceeds. The migS sequence, $211 \mathrm{~kb}$ clockwise of ori on the right replichore, facilitates sister ori separation away from mid-cell, but is not required for the final quarter positioning of sister ori regions (Yamaichi and Niki 2004). Furthermore, a segregation mechanism, like that proposed here, which moves the two leading strand templates (for example) toward the cell poles after initiation of replication will facilitate sister ori movement bidirectionally away from mid-cell.

The experimental data presented here lead to a testable model in which chromosome organization is directed by the proposed replication-segregation mechanism. A prediction of the model is that individual replichores occupy specific compartments that have a specific polarized "left-right-left-right" organization that is readily visualized in filaments and results from the heritable asymmetric ter segregation behavior observed. The model provides a platform for future studies that will test whether all chromosomal loci segregate asymmetrically through a mechanism that directs the leading and lagging strand templates of newly replicated sisters to distinct yet complementary addresses on either side of each developing sister nucleoid. Finally, we need to identify the processes and mechanisms that underlie the relationships between replication-segregation and chromosome organization described here, and their coupling to growth and cell division.

\section{Materials and methods}

\section{Bacterial strains and plasmids}

Strains used in this study were derivatives of E. coli K12 AB1157 (Bachmann 1972). The parental strains carrying 240 copies of the $1 a c O$ and tet $O$ arrays are described in Lau et al. (2003). The chromosomal ftsK-yfp gene was a gift of F.-X. Barre (CNRS CGM, Gif/Yvette, France). Plasmids were all derivatives of

Table 1. Bacteria and plasmids

\begin{tabular}{|c|c|c|}
\hline Name & Description & Other information \\
\hline $\begin{array}{l}\text { Bacteria } \\
\text { IL05 }\end{array}$ & $\begin{array}{l}\mathrm{AB} 1157 \\
\quad[\text { lacO240-Km]1801 } \\
\quad+ \\
{[\text { tetO240-Gm]3908 }}\end{array}$ & $\begin{array}{l}\text { tet } O \text { array } 15 \mathrm{~kb} \\
\text { counterclockwise } \\
\text { from oriC (oril), } \\
\text { lacO array } 210 \mathrm{~kb} \\
\text { clockwise from dif } \\
\text { (ter2) }\end{array}$ \\
\hline IL06 & $\begin{array}{l}\mathrm{AB} 1157 \\
{[\text { tet } 240-\mathrm{Km}] 1644+} \\
{[\text { lacO240-Gm]3908 }}\end{array}$ & $\begin{array}{l}\text { lacO array } 15 \mathrm{~kb} \\
\text { counterclockwise } \\
\text { from oriC, tetO } \\
\text { array } 50 \mathrm{~kb} \\
\text { clockwise from dif } \\
\text { (ter3). }\end{array}$ \\
\hline IL40 & $\begin{array}{l}\text { AB1157 } \\
\quad[\text { lacO240-Km]1380 } \\
\quad+ \\
\quad[\text { tetO240-Gm]1801 }\end{array}$ & $\begin{array}{l}\text { lacO array } 210 \mathrm{~kb} \\
\text { counterclockwise } \\
\text { from dif (ter4), tetO } \\
\text { array } 210 \mathrm{~kb} \\
\text { clockwise from dif } \\
\text { (ter2). }\end{array}$ \\
\hline IL2938 & $\begin{array}{l}\mathrm{AB} 1157 \\
\quad[\text { lacO } 240-\mathrm{Km}] 1644 \\
\quad+ \\
{[\text { tetO240-Gm]1801 }}\end{array}$ & $\begin{array}{l}\text { lacO array } 50 \mathrm{~kb} \\
\text { clockwise from dif, } \\
\text { tet } O \text { array } 210 \mathrm{~kb} \\
\text { clockwise from dif. }\end{array}$ \\
\hline $\begin{array}{l}\text { CP10 } \\
\text { CP11 }\end{array}$ & $\begin{array}{l}\text { IL05 ftsK-yfp }(\mathrm{Cm}) \\
\text { IL06 ftsK-yfp }(\mathrm{Cm})\end{array}$ & $\begin{array}{l}\text { Endogenous } \\
\text { expression of } \\
\text { FtsK-YFP fusion } \\
\text { protein. This } \\
\text { laboratory. }\end{array}$ \\
\hline $\begin{array}{c}\text { Plasmids } \\
\text { pWX1 }\end{array}$ & PBAD24-ftsZ-cfp (Ap) & $\begin{array}{l}\text { pBAD24 derivative } \\
\text { (Guzman et al. } \\
\text { 1995). }\end{array}$ \\
\hline $\begin{array}{l}\text { pCP8 } \\
\text { pCP12 } \\
\text { pWX6 } \\
\text { pWX9 }\end{array}$ & $\begin{array}{l}\mathbf{P} f t s K i-f t s Z-c f p(\mathrm{Ap}) \\
\mathbf{P} f t s K i-f t s Z-c f p+ \\
\text { lacI-yfp (Ap) } \\
\mathbf{P} f t s K i-1 a c I-c f p+ \\
\text { tetR-yfp (Ap) } \\
\mathbf{P} f t s K i-t e t R-c f p(\mathrm{Ap})\end{array}$ & $\begin{array}{l}\text { Multicopy pBR322 } \\
\text { derivative. } \\
\text { Constitutive low } \\
\text { expression of } \\
\text { proteins from a } \\
\text { weak promoter } \\
\text { within FtsK. This } \\
\text { laboratory. }\end{array}$ \\
\hline
\end{tabular}


pMB1. A weak constitutive promoter found in the C-terminal $1074 \mathrm{bp}$ of the ftsK gene was used for fluorescent protein expression. Bacteria and plasmids used are listed in Table 1.

\section{Microscopy and flow cytometry analysis}

Cells for microscopy were grown to $\mathrm{A}_{600}$ of $0.03-0.3$ in M9glycerol medium supplemented with required amino acids. In conditions where LacI or TetR were expressed, $0.5 \mathrm{mM}$ IPTG or $40 \mathrm{ng} / \mathrm{mL}$ anhydrotetracycline were also added to reduce repressor binding, without compromising focus formation. Cells were transferred to a $1.0 \%$ agarose layer containing phosphate-buffered saline for snapshot microscopy. In the case of time-lapse experiments, cells were transferred to a $1.0 \%$ agarose layer containing M9-glycerol medium when $\mathrm{A}_{600}$ reached 0.1. Nucleoids were visualized in $27 \%$ gelatin-containing minimal medium (Mason and Powelson 1956; H. Niki, pers. comm.) or after staining with $1 \mu \mathrm{g} / \mathrm{mL}$ DAPI. The growth of cells was followed on the slide at $37^{\circ} \mathrm{C}$ and images were captured every 5-30 min. Cells were visualized with a $100 \times$ objective on a Nikon Eclipse TE2000-U microscope, equipped with a Photometrics CoolSNAP HQ CCD camera and a temperature-controlled incubation chamber. The images were taken, analyzed, and processed by MetaMorph 6.2 and Adobe Photoshop.

\section{Biochemical procedures}

Established methods were used. Western blotting used a polyclonal antibody to E. coli FtsZ prepared in our laboratory.

\section{Acknowledgments}

We thank S. Filipe for his encouragement and discussions and for carrying out preliminary experiments that led to some of the work presented here, and $\mathrm{H}$. Niki for providing a protocol for the gelatin technique that allows visualization of nucleoids in the absence of fluorescent stains. We thank A. Whitely for assistance in flow cytometry. This work was supported by the Wellcome Trust. C.P. is supported by an EMBO long-term fellowship, and X.W. is in receipt of a Wellcome Trust Prize studentship.

\section{References}

Aarsman, M.E.G., Piette, A., Fraipont, C., Vinkenvleugel, T.M.F., Nguyen-Distèch, M., and den Blaauwen, T. 2005. Maturation of the Escherichia coli divisome occurs in two steps. Mol. Microbiol. 55: 1631-1645.

Aussel, L., Barre, F.-X., Aroyo, M., Stasiak, A., Stasiak, A.Z., and Sherratt D.J. 2002. FtsK is a DNA motor protein that activates chromosome dimer resolution by switching the catalytic state of the XerC and XerD recombinases. Cell 108: 195-205.

Bachmann, B.J. 1972. Pedigrees of some mutant strains of Escherichia coli K-12. Bacteriol. Rev. 36: 525-557.

Barre, F.-X. and Sherratt, D.J. 2005. Chromosome dimer resolution. In The bacterial chromosome (ed. N.P. Higgins), pp. 513-523. ASM Press, Washington, DC.

Bates, D. and Kleckner, N. 2005. Chromosome and replisome dynamics in E. coli: Loss of sister cohesion triggers global chromosome movement and mediates chromosome segregation. Cell 121: 899-911.

Ben-Yehuda, S., Rudner, R.Z., and Losick, R. 2003. RacA, a bacterial protein that anchors chromosomes to the cell poles. Science 299: 532-536.
Bernhardt, T.G. and de Boer, P.A.J. 2005. SlmA, a nucleoidassociated, FtsZ binding protein required for blocking septal ring assembly over chromosomes in E. coli. Mol. Cell 18: $555-564$.

Breier, A.M. and. Cozzarelli, N.R. 2004. Linear ordering and dynamic segregation of the bacterial chromosome. Proc. Natl. Acad. Sci. 101: 9175-9176.

den Blaauwen, T., Buddelmeijer, N., Aarsman, M.E.G., Hameete, C.M., and Nanninga, N. 1999. Timing of FtsZ assembly in Escherichia coli. J. Bacteriol. 181: 5167-5175.

Deng, S., Stein, R.A., and Higgins, N.P. 2004. Transcriptioninduced barriers to supercoil diffusion in the Salmonella typhimurium chromosome. Proc. Natl. Acad. Sci. 101: 33983403.

Draper, G.C. and Gober, J.W. 2002. Bacterial chromosome segregation. Ann. Rev. Microbiol. 56: 567-597.

Draper, G.C., McLennan, N., Begg, K., Masters, M., and Donachie, W.D. 1998. Only the N-terminal domain of FtsK functions in cell division. J. Bacteriol. 180: 4621-4627.

Dworkin, J. and Losick, R. 2002. Does RNA polymerase help drive chromosome segregation in bacteria? Proc. Natl. Acad. Sci. 99: 14089-14094.

Espeli, O. and Marians, K.J. 2004. Untangling intracellular DNA topology. Mol. Microbiol. 52: 925-931.

Espeli, O., Lee, C., and Marians, K.J. 2003. A physical and functional interaction between Escherichia coli FtsK and topoisomerase IV. J. Biol. Chem. 278: 44639-44644.

Fekete, R.A. and Chattoraj, D.K. 2005. A cis-acting sequence involved in chromosome segregation in Escherichia coli. Mol. Microbiol. 55: 175-183.

Gitai, Z., Dye, N.A., Reisenauer, A., Wachi, M., and Shapiro, L. 2005. MreB actin-mediated segregation of a specific region of a bacterial chromosome. Cell 120: 329-341.

Glaser, P., Sharpe, M.E., Raether, B., Perego, M., Ohlsen, K., and Errington, J. 1997. Dynamic mitotic-like behavior of a bacterial protein required for accurate chromosome partitioning. Genes \& Dev. 11: 1160-1168.

Gordon, G.S., Sitnikov, D. Webb, C.D., Teleman, A., Straight, A., Losick, R., Murray, A.W., and Wright, A. 1997. Chromosome and low copy plasmid segregation in E. coli: Visual evidence for distinct mechanisms. Cell 90: 1113-1121.

Guzman, L.M., Belin, D., Carson, M.J., and Beckwith, J. 1995. Tight regulation modulation and high level expression by vectors containing the arabinose $\mathrm{pBAD}$ promoter. I. Bacteriol. 177: 4121-4130.

Harry, E.J. 2001. Bacterial cell division: Regulating Z-ring formation. Mol. Microbiol. 40: 795-803.

Koppes, L.J., Woldringh, C.L., and Nanninga, N. 1999. Escherichia coli contains a DNA replication compartment in the cell center. Biochimie 81: 803-810.

Kruse, T., Moller-Jensen, J., Lobner-Olesen, A., and Gerdes, K. 2003. Dysfunctional MreB inhibits chromosome segregation in Escherichia coli. EMBO J. 22: 5283-5292.

Lau, I.F., Filipe S.R., Søballe, B., Økstad, O.-A., Barre, F.-X., and Sherratt, D.J. 2003. Spatial and temporal organisation of replicating Escherichia coli chromosomes. Mol. Microbiol. 49: 731-743.

Lemon, K.P. and Grossman, A.D. 2000. Movement of replicating DNA through a stationary replisome. Mol. Cell 6: 13211330 .

- 2001. The extrusion-capture model for chromosome portioning in bacteria. Genes \& Dev. 15: 2031-2041.

Lesterlin, C., Barre, F.-X., and Cornet, F. 2004. Genetic recombination and the cell cycle: What we have learned from chromosome dimers. Mol. Microbiol. 54: 1151-1160.

Li, Y., Sergueev, K., and Austin, S. 2002. The segregation of the 
Escherichia coli origin and terminus of replication. Mol. Microbiol. 46: 985-996.

Li, Y., Youngren, B., Sergueev, K., and Austin, S. 2003. Segregation of the Escherichia coli chromosome terminus. Mol. Microbiol. 50: 825-834.

Lin, D.C. and Grossman, A.D. 1998. Identification and characterization of a bacterial chromosome partitioning site. Cell 92: 675-685.

Margolin, W. 2001. Spatial regulation of cytokinesis in bacteria. Curr. Opin. Microbiol. 4: 647-652.

Mason, D.J. and Powelson, D.M. 1956. Nuclear division as observed in living bacteria by a new technique. J. Bacteriol. 71: 474-479.

Niki, H. and Hiraga, S. 1998. Polar localization of the replication origin and terminus in Escherichia coli nucleoids during chromosome partitioning. Genes \& Dev. 12: 1036-1045.

Niki, H., Yamaichi, Y., and Hiraga, S. 2000. Dynamic organization of chromosomal DNA in Escherichia coli. Genes \& Dev. 14: 212-223.

Postow, L., Hardy, C.D., Arsuaga, J., and Cozzarelli, N.R. 2004. Topological domain structure of the Escherichia coli chromosome. Genes \& Dev. 18: 1766-1779.

Recchia, G.D., Aroyo, M., Wolf, D., Blakely, G., and Sherratt, D.J. 1999. FtsK-dependent and -independent pathways of Xer site-specific recombination. EMBO J. 18: 5724-5734.

Rocha, E.P.C., Fralick, J., Vediyappan, G., Danchin, A., and Norris, V. 2003. A strand-specific model for chromosome segregation in bacteria. Mol. Microbiol. 49: 895-903.

Rodionov, O., Lobocka, M., and Yarmolinsky, M. 1999. Silencing of genes flanking the P1 plasmid centromere. Science 283: 546-549.

Roos, M., Van Geel, A., Aarsman, M., Veuskens, J., Woldringh, C., and Nanninga, N. 2001. The replicated ftsQZ and minB chromosomal regions of Escherichia coli segregate on average in line with nucleoid movement. Biochimie 81: 797802.

Sherratt, D.J. 2003. Bacterial chromosome dynamics. Science 301: 780-785.

Skarstad, K., Steen, H.B., and Boye, E. 1985. Escherichia coli DNA distributions measured by flow cytometry and compared with theoretical computer simulations. J. Bacteriol. 163: 661-668.

Soufo, H.J.D. and Graumann, P.L. 2004. Dynamic movement of actin-like proteins within bacterial cells. EMBO Rep. 5: 789 794.

Sun, Q. and Margolin, W. 1998. FtsZ dynamics during the division cycle of live Escherichia coli cells. I. Bacteriol. 180: 2050-2056.

. 2001 Influence of the nucleoid on placement of the FtsZ and MinE rings in Escherichia coli. J. Bacteriol. 183: 14131422.

Thanedar, S. and Margolin, W. 2004. FtsZ exhibits rapid movement and oscillation waves in helix-like patterns in Escherichia coli. Curr. Biol. 14: 1167-1173.

Valens, M., Penaud, S., Rossignol, M., Cornet, F., and Boccard, F. 2004. Macrodomain organization of the Escherichia coli chromosome. EMBO J. 23: 4330-4341.

Viollier, P.H. and Shapiro, L. 2004. Spatial complexity of mechanisms controlling a bacterial cell cycle. Curr. Opin. Microbiol. 7: 572-578.

Viollier, P.H., Thanbichler, M., McGrath, P.T., West, L., Meewan, M., McAdams, H.H., and Shapiro, L. 2004. Rapid and sequential movement of individual chromosomal loci to specific subcellular locations during bacterial DNA replication. Proc. Natl. Acad. Sci. 101: 9257-9262.

Woldringh, C.L. 2002. The role of co-transcriptional translation and protein translocation (transertion) in bacterial chromosome segregation. Mol. Microbiol. 45: 17-29.

Wu, L.J. and Errington, J. 2003. RacA and the Soj-Spo0J system combine to effect polar chromosome segregation in sporulating Bacillus subtilis. Mol. Microbiol. 49: 1463-1475.

2004. Coordination of cell division and chromosome segregation by a nucleoid occlusion protein in Bacillus subtilis. Cell 117: 915-925.

Yamaichi, Y. and Niki, H. 2004. MigS, a cis-acting site that affects bipolar positioning of oriC on the Escherichia coli chromosome. EMBO I. 23: 221-233.

$\mathrm{Yu}$, X.C., Weihe, E.K., and Margolin, W. 1998. Role of the C terminus of FtsK in Escherichia coli chromosome segregation. J. Bacteriol. 180: 6424-6428. 


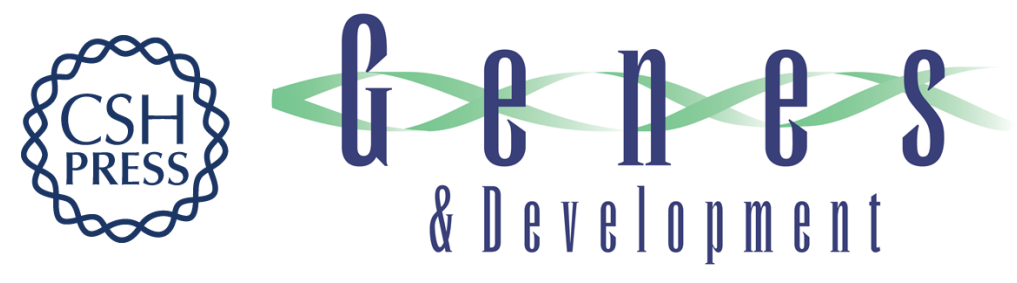

\section{Dancing around the divisome: asymmetric chromosome segregation in Escherichia coli}

Xindan Wang, Christophe Possoz and David J. Sherratt

Genes Dev. 2005, 19:

Access the most recent version at doi:10.1101/gad.345305

\section{Supplemental http://genesdev.cshlp.org/content/suppl/2005/09/20/19.19.2367.DC1 Material}

References This article cites 52 articles, 26 of which can be accessed free at: http://genesdev.cshlp.org/content/19/19/2367.full.html\#ref-list-1

\section{License}

Email Alerting

Receive free email alerts when new articles cite this article - sign up in the box at the top Service 